\title{
Modified submental platysmal adipomyofascial flap: is it a reliable alternate reconstructive option for small- to mid-sized defects especially in male patients with oral/oropharyngeal cancer?
}

\author{
Abhishek Singh ${ }^{1}$ (D) Anshu Chopra ${ }^{1} \cdot$ Shashank Chaudhary $^{1} \cdot$ Manikandan Venkatasubramaniyan $^{2} \cdot$ Kiran Joshi $^{3}$. \\ Mudit Agarwal $^{4}$
}

Received: 31 July 2020 / Accepted: 30 September 2020 / Published online: 9 October 2020

(c) Springer-Verlag GmbH Germany, part of Springer Nature 2020

\begin{abstract}
Purpose To introduce modified submental platysmal adipomyofascial flap as a new and viable hairless locoregional option for reconstruction of small- to mid-sized defects after ablative surgery in oral/oropharyngeal cancer patients keeping in mind the present pandemic situation.

Methods An observational retrospective study was conducted using modified submental platysmal adipomyofascial flap as a locoregional reconstructive option for both intraoral and oropharyngeal defects in early-stage oral/oropharyngeal cancer patients, from Jan 2016 to May 2020 in a tertiary care hospital. All patients in this study were male and the overall flap outcome was evaluated with post-operative follow-up.

Results Out of 18 patients, in 4 patients modified submental platysmal adipomyofascial flap was used as a combination of flaps for reconstruction. Six patients (33.33\%) underwent adjuvant radiation therapy/radiation chemotherapy. The long-term functions (speech and swallowing) and cosmetic outcomes were good in the majority of the patients. One patient (5.55\%) had pinhole oroantral fistula. No patient had any major flap failure.

Conclusion Modified submental platysmal adipomyofascial flap can be considered as a good alternative in male patients for reconstruction of small- to mid-sized oral cavity/oropharyngeal defects post-resection, especially during the prevailing pandemic crisis. It is an oncologically safe procedure with the major advantage of providing a hairless flap for oral cavity, tonsillar and BOT resection defects with lesser donor site morbidity.
\end{abstract}

Keywords Submental platysmal flap · Adipomyofascial flap $\cdot$ Submental flap $\cdot$ Locoregional flap

Abhishek Singh

dr.abhishekmds@gmail.com

Anshu Chopra

dr.anshuchopra@gmail.com

Shashank Chaudhary

chaudharyshashank747@gmail.com

Manikandan Venkatasubramaniyan drbel11988@gmail.com

Kiran Joshi

dr.kiranjoshi23@gmail.com

Mudit Agarwal

mudit1000@gmail.com

1 Head and Neck Oncology, RGCIRC, Rohini, Delhi, India

2 Surgical Oncology, RGCIRC, Rohini, Delhi, India

3 Head and Neck Unit II, RGCIRC, Rohini, Delhi, India

4 Head and Neck Surgical Oncology, RGCIRC, Rohini, Delhi, India

\section{Introduction}

Oral cavity cancer is the sixth most common cancer worldwide and comprises $30 \%$ of all head and neck cancers [1]. Most tumours of the oral cavity are squamous cell carcinomas (SCC), but other histological types such as minor salivary gland carcinomas, lymphomas and melanomas may also be seen rarely [2]. Surgery has been the mainstay for primary management of oral cavity cancer, while radiotherapy is offered post-operatively to patients at high risk for locoregional recurrence. The surgical resection entails removal of the tumour with a margin of at least $1-1.5 \mathrm{~cm}$. Neck dissection is simultaneously done for either clinically evident nodal disease, large primary tumours or tumours with a depth of invasion greater than $4 \mathrm{~mm} \mathrm{[2,3].} \mathrm{Cur-}$ rent recommendation of National Comprehensive Cancer Network (NCCN) guidelines for early stage oropharyngeal 
cancer (T1-T2, N0-N1) is either radiation therapy or surgery [4].

A variety of local flaps such as nasolabial flap, pectoralis major myocutaneous flap (PMMC), supraclavicular flap, sternocleidomastoid flap, platysma flap and various free flaps like the radial forearm and anterolateral thigh (ALT) flap are the main soft tissue reconstruction options available. However, all these options have their associated shortcomings. Reconstruction in the oral cavity and oropharyngeal region demands that the flap should be reliable, functionally and cosmetically acceptable with minimum donor site morbidity. Additionally, it must match the recipient site in terms of colour, texture and thickness [5]. The purpose of the study is the introduction of modified submental platysmal adipomyofascial flap (SPAF) as a new and viable hairless locoregional option for reconstruction of small- to mid-sized defects after ablative surgery in oral/oropharyngeal cancer patients, especially males, keeping in mind the present pandemic situation.

\section{Methodology}

A retrospective observational study using modified SPAF for reconstruction of small- to mid-sized defects in oral/oropharyngeal cancer patients from Jan 2016 to May 2020 was conducted in a tertiary cancer care hospital in Delhi, India. The study sample comprised 18 male patients who presented to our surgical oncology department with early-stage oral/ oropharyngeal cancer and underwent surgical resection with reconstruction of the resultant defects using the modified SPAF. Patients with clinical nodal stage more than $\mathrm{N}_{0}$ were excluded from the study. Females were also excluded from the study. Flap viability, complications, functional and cosmetic results were evaluated peri-operatively as well as postoperatively. All patients underwent metastatic workup before surgery and had biopsy-proven SCC. This study is in compliance with the Helsinki Declaration, and each subject in the project signed a detailed informed consent form. This study was also approved by the Institutional Hospital IRB. Informed consent was obtained from patients after full explanation of the surgical procedure, the likely outcome and the potential complications that may occur.

\section{Surgical technique}

Under general anaesthesia, the patient was placed in supine position with neck extended and head turned towards the opposite side. In all our patients with oral cavity primary, the primary lesion was resected first followed by raising the modified SPAF, except in one case in which modified SPAF was raised initially so that cheek flap could be raised through the angle of the mouth to facilitate resection of the buccal mucosa primary. In patients with early-stage oropharyngeal carcinoma, the modified SPAF was harvested first, followed by transoral robotic surgery (TORS) for the primary. Neck dissection was usually done after TORS.

Following the universal aseptic precautions, transverse cervical neck skin crease incision was placed with $2 \mathrm{~cm}$ extension across the midline in the majority of patients (Fig. 1). In patients who needed lip split incision for access to the primary, no transverse extension across midline was given. The skin flap of the neck was raised in a supraplatysmal plane, taking care not to perforate the skin. Superiorly, it was raised just above the inferior border of the mandible.

Modified SPAF based on submental vessels was raised with desired dimensions incorporating the ipsilateral anterior belly of digastric and mylohyoid muscles. First and foremost, the marginal mandibular nerve was identified and preserved. Facial vessels along with all the branches were traced and preserved. To facilitate removal of the submandibular gland, only those branches of the facial vessels supplying the gland were clipped and cut while preserving the hypoglossal and lingual nerves. The tendon of the anterior belly of the digastric as well as the mylohyoid muscle attachments was severed just above the hyoid bone over the surgeon's finger, which protected the underlying hypoglossal nerve and the lingual artery. Superiorly, the attachments of both the anterior belly of the digastric and mylohyoid muscle from the mandible were removed with periosteal elevator and bipolar cautery, taking care to avoid any inadvertent injury to the submental vessels.

We then approached from the contralateral anterior belly of the digastric muscle, dissecting the fascia off the muscle and raised the submental fat along with the platysma muscle, incorporating the ipsilateral anterior belly of the digastric into the flap. The mylohoid muscle was mostly cut at the

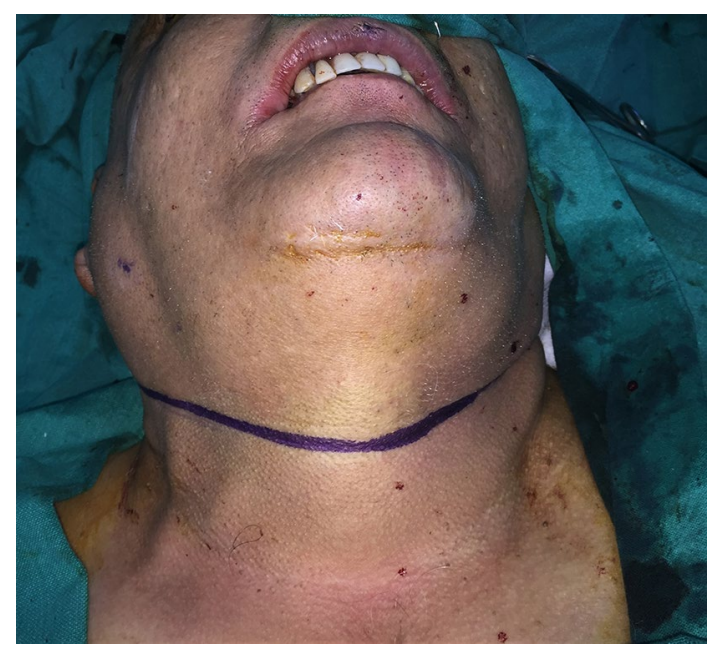

Fig. 1 Incision marking 


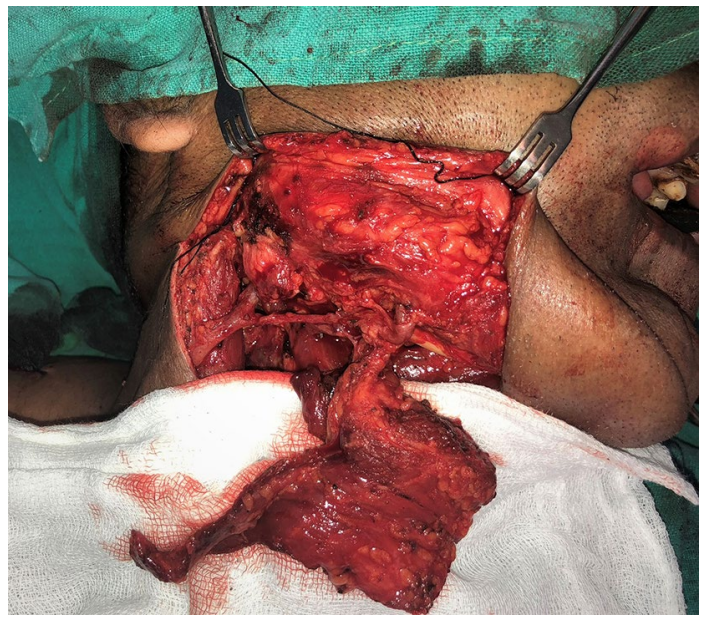

Fig. 2 Harvesting the submental platysmal adipomyofascial flap

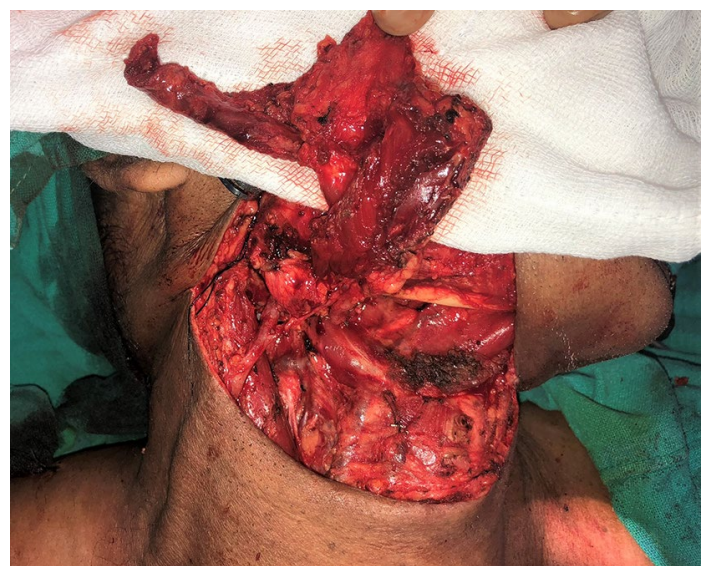

Fig. 3 Modified SPAF harvested medial border of the opposite anterior belly of the digastric muscle. The mylohyoid muscle can be raised partially or completely, depending on the bulk of the flap required for reconstruction (Fig. 2). The platysma muscle was also raised depending upon the size of the surgical defect. The inferior neck skin flap was also raised in a supraplatysmal plane and the platysma muscle was cut inferiorly, the maximum limit being at the level of the second transverse neck skin crease (Fig. 3). The remaining inferior neck skin flap was raised in a subplatysmal plane.

After the flap harvest was completed, the submental nodes were inspected, palpated and, whenever found, excised and sent for frozen section (FS). Flap insetting was only commenced, once the FS of level IA nodes was reported as negative. The harvested flap was tunnelled through the floor of the mouth, medial to the mandible into the oral cavity for reconstruction of tongue/palatal defects, whereas for buccal mucosa defects, it was delivered lateral to the mandible for final insetting into the surgical defect (Fig. 4). In cases where oropharyngeal defect reconstruction was needed, the modified SPAF was always tunnelled medial to the mandible via the ipsilateral floor of mouth.

\section{Results}

All 18 male patients underwent surgical resection and immediate reconstruction with modified SPAF (Table 1). The age of patients at presentation ranged from 31 to 76 years. The mean follow-up was around 18 months. The average dimension of the flap raised in total along with the platysma muscle was approximately $12 \times 6 \mathrm{~cm}$. The largest defect size reconstructed in our study was $5 \times 4 \mathrm{~cm}$. Simultaneous neck dissection was done in all patients. Ipsilateral supraomohyoid neck dissection (SOHND, levels I-III) was done in eight patients, four patients underwent ipsilateral modified neck dissection (MND, levels I-V) type III, five patients
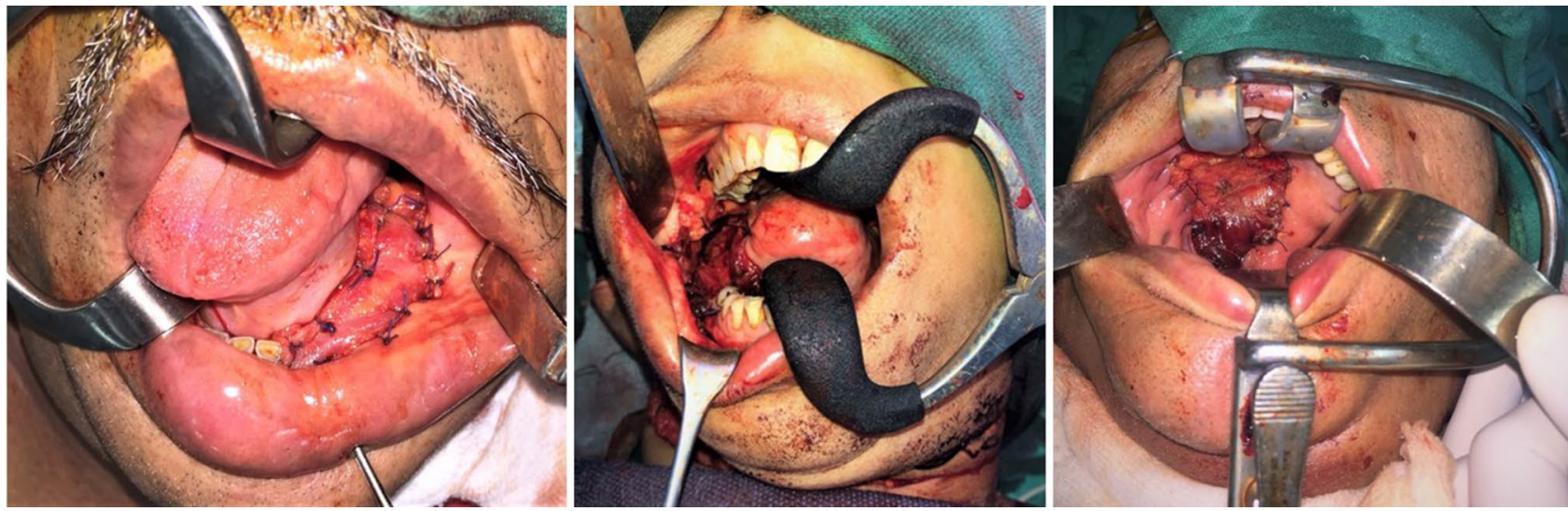

Fig. 4 Flap insetting into various surgical defects post-ablative surgery 


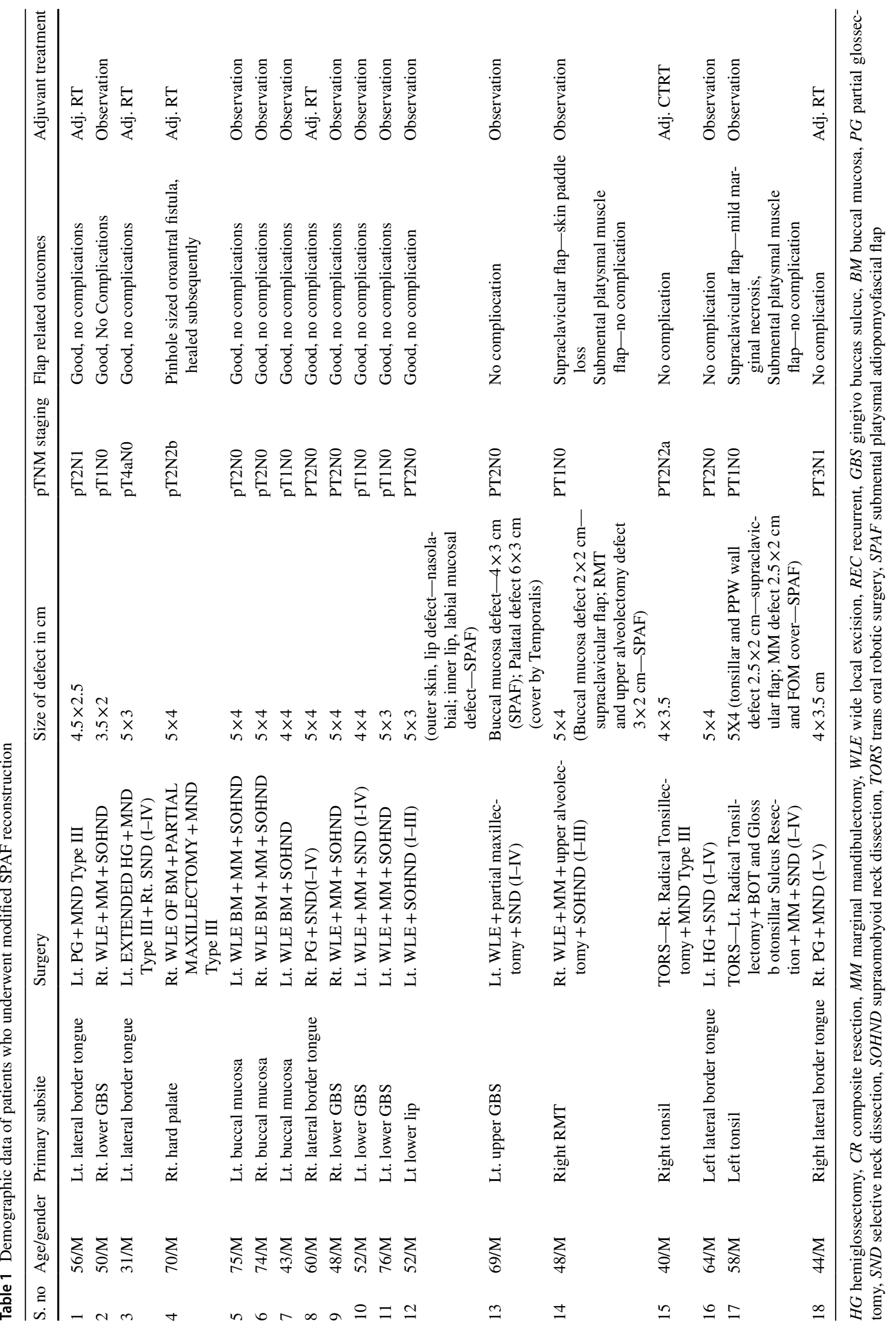


underwent ipsilateral selective neck dissection (SND, levels I-IV) and one patient underwent ipsilateral MND (levels I-V) type III and contralateral SND (levels I-IV). Neck dissection was always completed after the flap was harvested. All patients had intra-operative microscopic tumour-free margins assessed by frozen section.

All patients $(n=18,100 \%)$ had good uptake of modified SPAF with no major complication of flap necrosis. There was no skin necrosis of the donor neck area in any patient and the donor site healed uneventfully in all cases $(n=18$, $100 \%$ ).

Post-operative minor complication of a pinhole oroantral fistula was seen only in one patient $(n=1,5.55 \%)$, in whom the modified SPAF was used for palatal defect reconstruction. This was managed conservatively and, subsequently, it healed spontaneously.

None of the 18 patients had any complaints of intraoral hair growth on the flap, in the post-operative follow-up period. Five patients $(n=5,27.7 \%)$ required adjuvant radiation therapy (RT), while one patient $(n=1,5.55 \%)$ needed adjuvant radiation therapy chemotherapy (RTCT). Epithelisation of flap occurred within 3-4 weeks in all the cases. The long-term cosmetic outcomes and functions (speech and swallowing) were good in all the patients. In four patients, modified SPAF was used as an adjunct flap with another locoregional flap.

\section{Discussion}

In present times, the surgical defects created in patients with oral/oropharyngeal cancer, post-ablative surgery can be reconstructed using various reconstructive options. The most common choices are the microvascular free flaps, as they allow adequate reconstruction of the surgical defect. Microvascular reconstruction techniques increase the surgical time and complexity of the process and carry an inherent risk of vascular failure [6]. Gao et al. [7] in a study did an analysis on the cost-effectiveness of microvascular surgical reconstruction for head and neck defects after oncologic resection. In their study, they concluded that the cost of head and neck microvascular surgical reconstruction is higher than locoregional methods. There are several factors that contribute to higher cost such as prolonged operating time, need for sophisticated instrumentation, and prolonged hospitalization for flap monitoring. Various local flaps have also been used for reconstruction in head and neck cancer patients. The submental flap which was first described by Martin et al. has been widely used in head and neck reconstruction $[6,8]$.

The goal of reconstruction in head and neck cancer resection is to achieve adequate functionality and aesthetics by provision of like tissues with minimum morbidity to the donor site, thus maintaining quality of life of the patient. Submental flap's ready availability and the shorter operative time for its elevation offer obvious advantages over free tissue transfer [9].

The hair density of submental region in males is much higher in comparison to other parts of the body from where flaps for reconstruction can be harvested. This has been a major issue in the majority of cases in the post-operative period. In female patients, submental flap serves as a very good option for intraoral reconstruction as hair follicles are absent, whereas in male patients with thick beard, problems arise due to excessive hair growth in the reconstructed oral cavity. Post-operative adjuvant RT negates this problem to some extent. Katre et al. [10] reported their previous experience with submental flap, in which they experienced similar issue of hair-bearing skin in oral cavity in males, who did not receive adjuvant RT. These patients then had to undergo a second procedure of skin de-epithelialization or laser epilation at a later stage which further adds to the cost incurred by the patients.

In another study by Vural et al. [11] submental flap was used to reconstruct a defect in the oesophagus in a patient, and the donor site could not be closed primarily because of post-RT skin changes. A $3 \times 3 \mathrm{~cm}$ split-thickness skin graft harvested from the thigh was used to complete the closure of donor site. In the same study, in another case, submental flap was used to reconstruct the laryngeal defect, because the strap muscles were not suitable for repair of the defect (status post-RT). This patient had hair growth in the skin paddle of the submental flap which caused post-operative discomfort. Eventually, the patient underwent a revision surgery to replace the hair-bearing skin with a split-thickness skin graft.

De-epithelialized variant of submental flap has been previously described in literature to prevent the problem of hairbearing skin in the oral cavity. Rahpeyma et al. [12] introduced the orthograde submental flap (Pattel modification). The anterior belly of the digastric muscle and mylohyoid muscle in the pedicle half were included. In the nonpedicle part of the flap, the paddle was composed of platysma, subcutaneous fat tissue, and skin. The de-epithelialization process entailed sharp dissection of the skin just below the hair follicles after flap elevation. Secondary epithelialization on the raw surface of subcutaneous fat exposed to the oral cavity was completed post-operatively at 3 weeks $[12,13]$.

We have further modified our technique by leaving behind skin and subcutaneous tissue while taking the platysma, submental fat, ipsilateral digastric muscle and some part of mylohyoid muscle, comprising the bulk of the flap. The modified SPAF has several advantages. The necessity of primary closure after submental flap harvest using the conventional technique limits the size of the skin island that can be raised. In our modified SPAF, since the submental skin is left 
at its place and only the muscle and fat component is used, the need for mobilization of neck skin for primary closure is obviated. The donor site defect which is usually closed primarily in the conventional submental flap technique is associated with limited neck extension post-operatively. In modified SPAF, this is substituted by just the transverse neck skin crease incision, placed for neck dissection, which is then closed primarily with no loss of skin, and hence no associated difficulty in neck extension post-operatively. The donor site scar of the transverse neck skin crease incision in our study was cosmetically acceptable (Fig. 5). In our study, the majority of patients underwent transverse neck skin crease incision, whereas in one patient, it was further connected to the lip split incision at an angle of lip to provide access for the primary tumour resection. Closure of these neck incisions was done primarily and healed as an inconspicuous scar.

In our study, four patients underwent MND. This flap is usually advocated in a clinically N0 neck, but if any palpable or suspicious node is seen during SND/SOHND, it should be sent for frozen section and if positive the patient should undergo MND. As a protocol, we always assessed the submental fat as well as the nodal tissue for any clinically palpable or suspicious nodes by visual inspection and palpation, and if present, we always sent them for frozen section. We proceeded with modified SPAF reconstruction only if the submental node sent for FS was reported negative, whereas if reported positive, the flap was discarded and incorporated as part of the neck dissection specimen. Reconstruction was then done utilizing other locoregional flap options such as supraclavicular flap, lateral trapezius flap, pectorals major myocutaneous flap (PMMC) or the infrahyoid flap.

In a study by Wanj et al. [14] comprising 160 patients with primary T1-2 oral SCC, 127 were pN0, whereas 33 were $\mathrm{pN}+$. Submental island flap (SIF) failure was successful in $98.75 \%$ and flap failure was seen only in two patients. On follow-up, 18 patients had locoregional relapse of disease, of which 14 were $\mathrm{pN} 0$ and 4 were $\mathrm{pN}+$. The 5 -year recurrence free survival was $88.73 \%$ as compared to $86.93 \%$ for $\mathrm{pN} 0$ and $\mathrm{pN}+$ groups, respectively $(p=0.847)$. The $\mathrm{pN}+$ patients had poorer prognosis than $\mathrm{pN} 0$ patients [5 years overall survival, $66.35 \%$ compared to $91.10 \%$ respectively $(p=0.005)]$. Multivariate analyses in this study showed no independent predictor responsible for locoregional recurrence, but $\mathrm{pN}+$ status was predictive for poor prognosis $(p=0.03)$. SIF did not increase the risk of locoregional recurrence in patients with $\mathrm{pN}+\mathrm{T} 1-2$ OSCC compared to those with pN0 T1-2 OSCC if careful neck dissection with appropriate post-operative adjuvant treatment was done. In our study, none of the patients had presented with any local or regional nodal recurrence on routine follow-up.

To overcome the problem of hair growth on flaps harvested from hair growing areas of the body, various options such as second surgery, radiation, de-epithelialization and laser epilation have been described in the past $[15,16]$. In a study by Rahpeyma et al. [17] three ways had been used for management of intraoral hairs: radiotherapy, second surgery and flap de-epithelialization. In nine male patients who needed radiotherapy after surgery (because of the malignant mucosal lesions), the main role of adjuvant RT was for
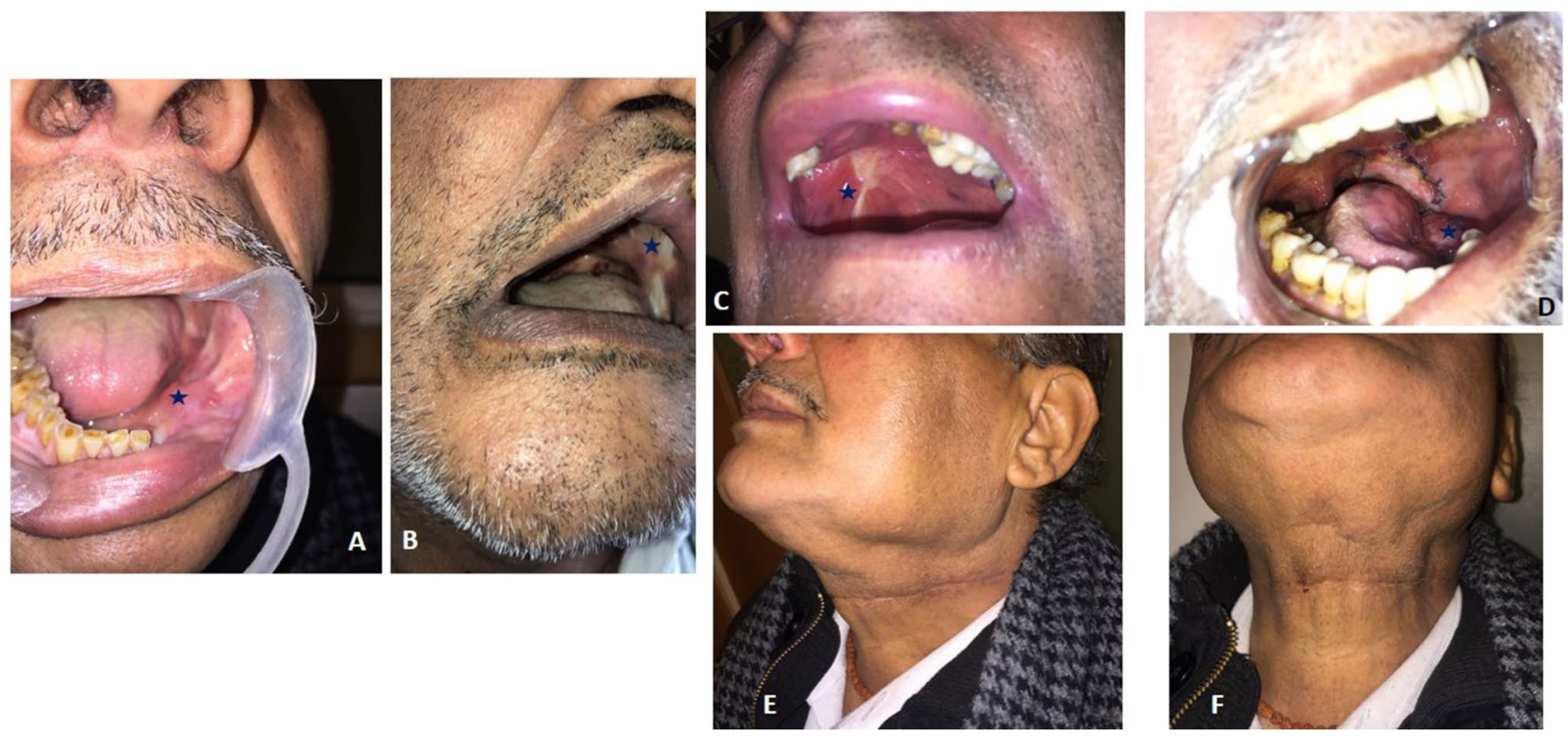

Fig. 5 Post-operative picture of modified SPAF (a-c), supraclavicular and modified SPAF combination (d), post-op image and post-op scar image (e, f). (Note: marking denotes the modified SPAF) 
controlling the local recurrence of disease but was additive for the management of transferred hair in the oral cavity. In two patients with verrucous carcinoma of the hard palate and alveolar process, a second surgery was done to eliminate the intraoral hair growth on the flap, whereas in the remaining male patients (52\%), flap de-epithelialization was done during the surgery for removing unwanted hair [17].

The submental flap has a long and reliable vascular pedicle, which provides a pedicle length up to $8 \mathrm{~cm}$, with an average of 2-mm arterial diameter. Although the largest skin paddle used was $14 \times 7 \mathrm{~cm}$ according to the English literature, it has been proposed that a skin paddle up to $18 \times 7 \mathrm{~cm}$ is possible, depending on the laxity of the submental skin [10].

Reconstruction of palatal defects has traditionally been accomplished with prosthetic obturator or local palatal flaps. Prosthetic obturators can present a difficult problem for the elderly. Such patients frequently have a compromise in manual dexterity and/or poor eyesight, making management and placement of prosthesis difficult. An ideal reconstructive technique for the rehabilitation of palatal defects is one that can achieve permanent separation of the oral and nasal cavities without precluding the retention of a tissueborne denture [18]. In a case report by Genden et al. [18], they have used submental flap for the repair of a hard palate defect measuring $5 \times 7 \mathrm{~cm}$ with communication to the ipsilateral antrum. A submental flap with similar dimensions was raised for reconstruction of the palatomaxillary defect. Such a large size flap cannot be raised if skin laxity is not present for primary closure of the defect. If tight closure is accomplished, then the patient will have severe limitation of extension of neck. The flap was passed through a subcutaneous tunnel and used to reconstruct the intraoral defect and partition the oral-antral communication.

In our study, one patient had reconstruction of the hard palate defect with modified SPAF with a defect size of $5 \times 3 \mathrm{~cm}$. Epithelization occurred within 3 weeks, but this patient had a pinhole sized oroantral fistula which was managed conservatively and healed subsequently. The reach of this flap can also be further extended by ligating and dividing the facial artery and vein at the inferior border of mandible while preserving the marginal mandibular nerve. This manoeuvre allows an additional reach of $2-3 \mathrm{~cm}$.

Modified SPAF can also be used as an adjunct flap with other locoregional flaps. In our study, we have done this in four patients. In one patient, an almost complete lower lip defect was reconstructed using a combination of the left nasolabial flap for the outer skin and lip reconstruction, whereas the modified SPAF was used for reconstructing the inner labial mucosal and lip defect. This combination technique additionally helped in achieving good vertical height and neolip competence. In another patient, after wide local excision (WLE) and partial maxillectomy, modified SPAF was used in combination with left temporalis flap for reconstruction of the buccal mucosal defect $(4 \times 3 \mathrm{~cm})$ and temporalis was used for providing palatal cover. Modified SPAF was also used as a combination flap with supraclavicular flap in two patients. In one patient, the modified SPAF was used to reconstruct the RMT, marginal mandibulectomy and upper alveolectomy defect, whereas the supraclavicular flap was used to reconstruct the buccal mucosal defect. The reason for using modified SPAF in this patient as an adjunct was based on our experience with the supraclavicular flap. If one chooses to reconstruct such a surgical defect with only the supraclavicular flap, then the supraclavicular flap is usually delivered lateral to the remnant mandibular bone. Additionally, to provide cover over the marginal mandibulectomy site and RMT defect site, the RMT hitching suture is taken, which can cause undue traction on the flap leading to flap congestion and failure. Hence in this patient, the modified SPAF helped in negating this particular problem and provided better soft tissue cover over the remnant native mandibular bone, RMT defect and the upper alveolectomy defect. The supraclavicular flap, which was used for buccal mucosal defect reconstruction, had skin paddle loss in the post-operative setting and was managed with debridement and secondary healing, whereas modified SPAF had no flap loss complication. In another patient, both supraclavicular flap and modified SPAF were delivered medial to the mandible. Modified SPAF was used for providing soft tissue cover over the remnant native mandibular bone (post-marginal mandibulectomy) and ipsilateral floor of mouth (FOM), whereas the supraclavicular flap was used to reconstruct the posterior pharyngeal wall and left tonsillar fossa defect, keeping in mind the problem associated with supraclavicular flap especially when providing cover for RMT and marginal mandibulectomy defect over the remnant mandible as described earlier.

Overall, flap outcome in all the cases have been appreciably good with no major morbidity due to the flap. Modified SPAF is a good alternative option for reconstruction of small- to medium-sized oral cavity and oropharyngeal ablative defects, especially in male cancer patients. It is an oncologically safe procedure with the primary advantage of providing a hairless flap for oral cavity/oropharynx, with lesser donor site morbidity. This flap offers greater advantage, especially in T1/T2 lesion patients who are less likely to receive adjuvant RT, as it overcomes the major disadvantage of hair growth intraorally as well as in the oropharynx which is associated with the conventional submental flap. Another advantage is negating the need for additional circumferential incision in the submental area, as modified SPAF flap can be harvested utilizing the same neck skin crease incision placed for the neck dissection. This additionally helps in better extension of neck as opposed to patients 
who have undergone primary closure of donor site when using the conventional approach for submental flap harvest.

In comparison to free microvascular flaps, it is more economical to the patient with less operative period and hospital stay. A meta-analysis by $\mathrm{Hu}$ et al. [19] concluded that submental island flap was associated with less operative time, shorter hospitalization, fewer peri-operative complications, and potentially similar disease recurrence rates compared to free tissue transfer for the reconstruction of oral cavity ablative defects. Additionally amongst the two cohorts, there was no difference in the rate of flap loss.

Modified SPAF can also be combined with other locoregional flaps as an adjunct flap for reconstruction of surgical defects. This flap can also be used in select subset of females, who in addition to the neck incision scar do not want the extended scar due to the conventional submental flap harvest incision, though we have not included any female patients in our present study. A shortcoming of this study is the relatively small sample size. Further comparative study needs to be done with patients undergoing conventional submental flap, especially for the quantitative assessment and difference of neck extension limitation.

\section{Conclusion}

Our study concludes that this flap is a reliable and good reconstructive option in male patients, especially for smallto medium-sized oral/oropharyngeal defects. In the present scenario of COVID-19 pandemic crisis, alternative locoregional options for flap reconstruction in the head and neck might be more suitable as compared to free flap reconstruction due to emphasis on less operative time, shorter flap harvest time and less post-op complications and shorter hospital stay. Modified SPAF could be an alternate addition to the armamentarium of locoregonal flaps for the reconstructive/head and neck surgeons to be applied in head and neck reconstruction in the select subset of patients with minimal donor site morbidity in the prevailing pandemic times.

Funding Nil.

\section{Compliance with ethical standards}

Ethical approval Obtained from the Institutional Scientific Committee.

Informed consent Patient consents were taken.

\section{References}

1. Jemal A, Siegel R, Ward E et al (2006) Cancer statistics, 2006. CA Cancer J Clin 56(2):106-130. https://doi.org/10.3322/canjc lin.56.2.106
2. Amin AA, Sakkary MA, Khalil AA, Rifaat MA, Zayed SB (2011) The submental flap for oral cavity reconstruction: extended indications and technical refinements. Head Neck Oncol 3(1):51. https://doi.org/10.1186/1758-3284-3-51

3. Chen W, Dong Z, Cao G, Liu B, Meng Z, Zhang S (2013) Reconstruction of anterior floor of mouth defects by the local mandible myofascial flap following cancer ablation: a pilot study. J Plast Reconstr Aesthetic Surg 66(9):1238-1242. https ://doi.org/10.1016/j.bjps.2013.04.008

4. Pfister DG, Spencer S, Adelstein D, Adkins D, Anzai Y, Brizel DM, Bruce JY, Busse PM, Caudell JJ, Cmelak AJ, Colevas AD (2020) Head and neck cancers, version 2.2020, NCCN clinical practice guidelines in oncology. J Natl Compr Cancer Netw 18(7):873-898

5. Sebastian P, Thomas S, Varghese BT, Iype EM, Balagopal PG, Mathew PC (2008) The submental island flap for reconstruction of intraoral defects in oral cancer patients. Oral Oncol 44(11):1014-1018. https://doi.org/10.1016/j.oraloncolo gy.2008.02.013

6. Abouchadi A, Capon-Degardin N, Patenôtre P, Martinot-Duquennoy V, Pellerin P (2007) The submental flap in facial reconstruction: advantages and limitations. J Oral Maxillofac Surg 65(5):863-869. https://doi.org/10.1016/j.joms.2006.05.063

7. Gao LL, Basta M, Kanchwala SK, Serletti JM, Low DW, Wu LC (2017) Cost-effectiveness of microsurgical reconstruction for head and neck defects after oncologic resection. Head Neck 39(3):541-547. https://doi.org/10.1002/hed.24644

8. Martin D, Baudet J, Mondie JM, Peri G (1990) The submental island skin flap. A surgical protocol. Prospects of use. Ann Chir Plast Esthétique 35(6):480-484

9. Thomas S, Varghese BT, Ganesh SA et al (2016) Oncological safety of submental artery island flap in oral reconstructionanalysis of 229 cases. Indian J Surg Oncol 7(4):420-424. https:// doi.org/10.1007/s13193-016-0532-2

10. Katre MI, Keche P, Gaikwad A, Deshmukh S (2017) Versatility of the submental island flap in head and reconstruction. Int J Otorhinolaryngol Head Neck Surg 3(4):1072-1076

11. Vural E, Suen JY (2000) The submental island flap in head and neck reconstruction. Head Neck 22(6):572-578

12. Rahpeyma A, Khajehahmadi S (2014) Onlay bone grafting simultaneous with facial soft tissue augmentation in a hemifacial microsomia patient using de-epithelialized orthograde submental flap: a technical note. Ann Stomatol (Roma) 5(1):30-33

13. Rahpeyma A, Khajehahmadi S (2013) Oral reconstruction with submental flap. Ann Maxillofac Surg 3(2):144-147. https://doi. org/10.4103/2231-0746.119224

14. Wang J, Tan Y, Shen Y, Lv M, Li J, Sun J (2020) Oncological safety of submental island flap for reconstruction of pathologically node-negative and node-positive T1-2 oral squamous cell carcinoma-related defects: a retrospective study and comparison of outcomes. Oral Oncol 102:104507. https://doi.org/10.1016/j. oraloncology.2019.104507

15. Freites-Martinez A, Shapiro J, van den Hurk C, Goldfarb S, Jimenez JJ, Rossi AM, Paus R, Lacouture ME (2019) Hair disorders in cancer survivors. J Am Acad Dermatol 80(5):1199-1213. https:// doi.org/10.1016/j.jaad.2018.03.056 (Epub 2018 Apr 14)

16. Shields BE, Moye MS, Bayon R, Sperry SM, Wanat KA (2018) A hairy situation: laser hair removal after oral reconstruction. Ann Otol Rhinol Laryngol 127(3):205-208. https://doi. org/10.1177/0003489417750930 (Epub 2017 Dec 31)

17. Rahpeyma A, Khajehahmadi S (2017) A protocol for management of the hairs problem in oral cavity reconstruction by submental flap. J Maxillofac Oral Surg 16(1):108-112. https://doi. org/10.1007/s12663-016-0925-3

18. Genden EM, Buchbinder D, Urken ML (2004) The submental island flap for palatal reconstruction: a novel technique. $\mathrm{J}$ 
Oral Maxillofac Surg 62(3):387-390. https://doi.org/10.1016/j. joms.2003.06.009

19. Hu S, Fan C, Pecchia B, Rosenberg JD (2020) Submental island flap vs free tissue transfer in oral cavity reconstruction: systematic review and meta-analysis. Head Neck. https://doi.org/10.1002/ hed.26121
Publisher's Note Springer Nature remains neutral with regard to jurisdictional claims in published maps and institutional affiliations. 